\title{
DA CARTOGRAFIA ANALÓGICA À NEOCARTOGRAFIA: NOSSOS MAPAS NUNCA MAIS SERÃO OS MESMOS?
}

\author{
Maria Isabel Castreghini de Freitas ${ }^{1}$
}

Resumo: Esse artigo objetiva realizar uma reflexão sobre os caminhos da cartografia apoiada nas tecnologias, tomando por base o histórico recente da ciência cartográfica no Brasil, bem como apresentar as possibilidades da Neocartografia no presente e no futuro, considerando a realidade globalizada e as tendências de uma cartografia apoiada nas mídias e na internet. $O$ texto esta estruturado em duas partes: a primeira trata de uma revisão da história recente da cartografia no Mundo e no Brasil, destacando os principais eventos que transformaram nossa forma de produzir mapas nas últimas décadas até os dias atuais; a segunda parte trata de uma reflexão sobre os rumos da Cartografia com o advento de tecnologias de comunicação e posicionamento como os sistemas de computacionais $e$ internet de alta velocidade, contribuindo para o surgimento de uma nova geração de cartógrafos e usuários de mapas, apoiados em recursos tecnológicos para a produção e uso de mapas de maneira dinâmica e instantânea. Pretende-se fomentar a discussão sobre o que é imprescindível e imutável na ciência cartográfica, nesse novo relacionamento com os mapas.

Palavras-chaves: história da cartografia; internet; neocartografia; tecnologia

From the analogical cartography to the neocartography: will our maps ever be the same?

Abstract: This article aims to carry out a reflection on the paths of technologies supported cartography, based on the recent history of the cartographic science in Brazil, as well as to present the possibilities of the neocartography both presently and in the future, considering the globalized reality and trends of a cartography based on media and internet. The text is divided into two parts: the first one is a review of the recent history of cartography in the World and in Brazil, highlighting the major events that have transformed our way of producing maps in recent decades to the present day; the second part is a reflection on the course of cartography with the advent of communication and positioning technologies such as the computer systems and high-speed internet, contributing to the emergence of a new generation of cartographers and map users, supported by technological resources for the production and use of maps dynamically and instantly. It is intended to promote discussion on what is essential and unchanging in cartographic science, as well as what probably will fall into disuse in this new relationship with the maps.

Keywords: history of cartography; internet; neocartography; technology

\footnotetext{
${ }^{1}$ Engenheira Cartógrafa pela UNESP/Presidente Prudente SP, Mestre pela UFPR/Curitiba PR, Doutora em Engenharia pela POLI-USP e Livre Docente pela UNESP/Rio Claro SP. Email: ifreitas@rc.unesp.br
} 


\section{INTRODUÇÃO}

Ao olharmos para a história da Cartografia ao longo do tempo, observamos sua evolução em sintonia com os grandes avanços tecnológicos da humanidade.

Esse artigo objetiva realizar uma reflexão sobre os caminhos da cartografia apoiada nas tecnologias, tomando por base o histórico recente da ciência cartográfica no Brasil, bem como apresentar as possibilidades da Neocartografia no presente e no futuro, considerando a realidade globalizada e as tendências de uma cartografia apoiada nas mídias e na internet. Além do caráter reflexivo, por meio de resgate histórico da Cartografia considerados alguns fragmentos de registros históricos que influenciaram a sua construção como ciência e como técnica, bem como as transformações até os dias atuais, pretende-se também apresentar alguns resultados de pesquisas realizadas em textos selecionados, na busca de entender os caminhos teórico-metodológicos que vem sendo trilhado por pesquisadores que são produtores e usuários de mapas não convencionais.

\section{Fragmentos Históricos da Cartografia Analógica e Digital}

Os avanços tecnológicos nos diferentes momentos históricos pelos quais passou o homem ditam os passos da Cartografia em termos de orientação na Terra e representação do espaço e de fenômenos naturais ou produzidos pelo Homem.

Como exemplos podemos considerar fragmentos da história da Cartografia no mundo e no Brasil, considerando eventos marcantes como a invenção de instrumentos de medição como bússolas, astrolábios, teodolitos, balões, aeronaves e câmeras fotográficas. Resguardadas as dimensões dos impactos dos eventos em suas épocas, não se entende Cartografia sem tecnologia. Isso pode ser constatado desde os primitivos registros de representações cartográficas fazendo uso de pigmentos coloridos em aglomerados rochosos, modelagem em argila como os mapas babilônicos, trançados de folhas de palmeiras como os dos nativos das Ilhas Marshall da Austrália, dentre tantos outros registros cartográficos da humanidade, indicando a constante busca do homem por instrumentos que facilitassem sua sobrevivência, comunicação, orientação e transporte.

Os anos após a Segunda Guerra Mundial apresentaram profundas transformações em nossa sociedade numa perspectiva cada vez mais global de diminuição das distancias entre os 
continentes, fruto dos avanços nas tecnologias de transporte e comunicação associadas ao primeiros ensaios para o desenvolvimento do computador, que trouxeram importantes alterações nas técnicas de mapeamento e nos produtos cartográficos.

Dentre os marcos históricos da Cartografia destacamos o uso associado de imagens fotográficas disponíveis após os experimentos bem sucedidos de fixação da fotografia por Joseph Niépce (1765 - 1836) e por Louis Dagerre (1787 - 1851) no início do século XIX, em combinação com as técnicas de visualização das imagens estereoscópicas tridimensionais ${ }^{2}$ já conhecidas e os balões, seguidos das aeronaves inventadas no inicio do século XX, permitiram as tomadas fotográficas aéreas que deram os primeiros passos para o mapeamento sistemático, tendo como produtos mapas topográficos de grandes extensões do território. Essa combinação de inventos revolucionários para a humanidade permitiram à Cartografia importantes transformações que refletiram em ganho de qualidade e precisão.

Os estudos de Cartografia dos anos de 1960 até 1980, com o advento do computador e sua disponibilização para fins civis, juntamente com a rápida evolução dos sistemas computacionais e o aprimoramento dos computadores e seus componentes, correspondeu à disseminação de iniciativas predominantemente de grupos de professores universitários e centros de pesquisa em todo o mundo dedicados a desenvolver rotinas para transformar os mapas analógicos em digitais com vistas à analise espacial. Um exemplo precursor é o de Roger Tomlinson (1933 - 2014) que no inicio dos anos de 1960 desenvolveu o primeiro Sistema de Informação Geográfica (SIG) para o Inventário das Terras do Canadá, que serviu de ponto de partida para inúmeras experiências que frutificaram em diferentes sistemas comercializados em nível mundial, bem como em outros de desenvolvimento e caráter acadêmico.

No Brasil o Grupo de Organização da Comissão Nacional de Atividades Espaciais (GOCNAE), instituído pelo Governo Federal no inicio dos anos de 1960, permitiu os primeiros passos para a criação do Instituto Nacional de Pesquisas Espaciais em 1971, órgão voltado para o desenvolvimento de pesquisa voltada para os estudos espaciais, seja por meio de satélites meteorológicos, de comunicação ou de observação da Terra. Um marco para a Cartografia nacional foi a implantação, nos anos de 1972 - 1973, da estação de recepção de dados de satélite de sensoriamento remoto em Cuiabá (MT), que permitiu ao Brasil pioneirismo na

\footnotetext{
${ }^{2}$ Por exemplo a técnica do Anaglifo que utiliza cores complementares nas imagens superpostas, tomadas de pontos de vista diferentes, que permitem a visualização 3D por meio de óculos com filtros que separam as 2 imagens, formando a imagem estereoscópica no cérebro do usuário.
} 
recepção e distribuição de imagens orbitais oriundas dos satélites Landsat (EUA) na América Latina. Tais ações permitiram que a Cartografia e o Sensoriamento Remoto nacionais tivessem avanços a largos passos, apoiados em tecnologias de ponta no que se refere a equipamentos e programas computacionais de mapeamento apoiado em imagens orbitais e processamento digital de imagens (CAMARA, 2011).

Os governos militares da época priorizaram os mapeamentos sistemáticos do país, período florescente para a Cartografia por meio de projetos de mapeamento liderados e normatizados pela Diretoria do Serviço Geográfico do Exército (DSG) tendo como mote a integração nacional, cujo slogan era Integrar para não Entregar. Muitos dos documentos cartográficos que temos acesso e utilizamos nos dias atuais são oriundos deste período quando tínhamos a intensificação da produção cartográfica sistemática no país, em diferentes escalas.

Nos anos de 1980 e 1990 a abertura comercial brasileira, com a redução das alíquotas de importação, trouxe avanços e a internacionalização de nossa sociedade, bem como maior acesso a tecnologia que se desenvolvia a largos passos no primeiro mundo. O capitalismo se fortalece, se transforma e nos transforma, formando grandes complexos corporativos que detém o controle da indústria e do comercio globais, transformando a Terra numa imensa aldeia de produtos cada vez mais sofisticados e menos diversificados. Esses novos movimentos se refletem na Cartografia e nas técnicas de mapeamento e de analise do meio urbano e rural.

Em termos de programas computacionais tiveram grande repercussão na época os programas específicos para desenho auxiliados por computador (Computer-Aided Design CAD), com destaque para americano AUTOCAD, lançado em 1982, que começaram a ser disseminados de forma acelerada. Neste período, nos Estados Unidos, foi lançada a primeira versão do SIG ARC INFO pela ESRI ${ }^{3}$, pacote de programas modulares, no início programado em FORTRAN para ser executado em minicomputadores.

Paralelamente e em sintonia com os avanços mundiais, ocorreram iniciativas para implantação de sistemas de Cartografia Digital no Brasil como, por exemplo, o MAXICAD, da Empresa Maxidata, dentre tantos outros exemplos que poderiam ser citados.

Mais de uma década antes, no ano de 1973, o Departamento de Defesa dos EUA cria o Sistema de Posicionamento Global (GPS), que transformaria radicalmente a forma de se

\footnotetext{
${ }^{3}$ Environmental System Research Institute (ESRI) - EUA
} 
fazer cartografia, garantindo locação precisa de qualquer posição na superfície terrestre. Foi também nesta década, em 1975 que Bill Gates e Paul Allen fundaram a Microsoft, cujo objetivo inicial era desenvolver e comercializar interpretadores da linguagem computacional BASIC e transformando-se em uma das empresas de tecnologia de destaque mundial, com alto capital resultante de desenvolvimento computacional em equipamentos e programas computacionais e também com grandes investimentos em pesquisa e desenvolvimento.

Os SIG que, numa primeira geração tinham formato matricial, foram se transformando, conforme os recursos tecnológicos da computação gráfica se aprimoravam, em sistemas vetoriais.

Dentre os Sistemas de Informação Geográfica (SIG) de uso corrente no Brasil, um dos precursores foi SITIM/SGI, composto do SITIM (Sistema de Tratamento de Imagens) no ambiente MS-DOS do INPE lançado em 1986, com ferramentas poderosas de processamento de imagens. Posteriormente ocorreu a integração com o Sistema de Informação Geográfica, denominado SGI, que integrava imagens aos mapas.

Em 1991 teve inicio o desenvolvimento do SPRING baseado num pioneiro modelo de dados que combinava informações alfanuméricas à geo-objetos, numa perspectiva de SIG integrado ao Banco de Dados, antecipando os avanços que os SIG comerciais só alcançariam anos mais tarde. Surgem assim os SIG integrados, cuja base é um robusto Banco de Dados, ampliando em muito as possibilidades de integração de informação relacionada a Dados Socioeconômicos e ambientais às bases cartográficas.

Outro importante SIG nacional é o Sistema de Análise Geo-Ambiental - SAGA/UFRJ, implantado em 1983, no Departamento de Geografia da UFRJ, pelo Prof.Dr. Jorge Xavier da Silva, que desde então tem desenvolvido grande volume de pesquisas, trabalhos e publicações acadêmicas do uso de SIG voltado para os estudos ambientais. Vinculado à Universidade Pública também se destacou no inicio dos anos 1990 o Geo-Inf-Map, SIG desenvolvido pelo Prof. Dr. Amandio Luiz de Almeida Teixeira no Departamento de Cartografia e Análise da Informação Geográfica da UNESP - Rio Claro SP.

Outro programa, agora de caráter comercial, amplamente utilizado no Brasil nesse período foi o IDRISI, desenvolvido pela Clark University - EUA, cuja versão DOS 3.2 lançada em 1989 e a versão para Windows disponível a partir de 1996.

Tais sistemas de Sensoriamento Remoto e SIG permitiram a disseminação da produção cartográfica em termos nacionais, principalmente por meio de ações das universidades, 
institutos de pesquisa e empresas privadas em parceria com órgãos dos governos municipais, estaduais e federais. Além disso, possibilitaram a formação acadêmica de uma geração de profissionais brasileiros respondem pelos aprimoramentos das técnicas de processamento digital de imagens e de sistemas de informação geográfica, tanto no meio empresarial quanto acadêmico.

Os anos de 1990 e 2000 dão oportunidade de desenvolvimento de sistemas de gerenciamento de bancos de dados extensíveis, orientados a objetos, que tem predominado na atualidade. Ocorre, neste período a grande proliferação de SIG comerciais, com recursos sofisticados. Nesse mesmo compasso, prolifera-se a perspectiva dos sistemas operacionais com códigos-fonte abertos, como o Linux, lançado em 1991.

Durante esta evolução surge, em especial no meio acadêmico, o desenvolvimento de sistemas de cartografia digital e SIG abertos, disponíveis na internet, aos quais qualquer usuário tem acesso sem pagar por licenças de uso, como tradicionalmente ocorre com os sistemas comerciais. A proposta de sistemas abertos, de acesso gratuito aos usuários, faz aumentar vertiginosamente os projetos cartográficos apoiados nesta tecnologia.

No mesmo ritmo o sensoriamento remoto evolui no período, disponibilizando sensores de alta resolução como, por exemplo o IKONOS, da empresa americana Space Imaging, lançado em 1999, produzindo imagens de resolução de 1 a 4m. Outro exemplo é o Quickbird, da empresa Digital Globe, lançado em 2001 e com resolução de $60 \mathrm{~cm}$ a 3m.

No que concerne à modelagem do terreno utilizando o computador existem registros do desenvolvimento de modelos digitais do terreno (MDT) por Charles L. Miller e colaboradores no Instituto de Tecnologia de Massachusetts entre 1955 a 1960.

Os modelos digitais do terreno (MDT) são definidos como arranjos ordenados de números, que reproduzem a distribuição espacial das características do terreno (DOYLE, 1978).

Esses modelos se aprimoraram desde então, como por exemplo o SURFER ${ }^{4}$, com grande difusão no Brasil. Em fevereiro de 2000, com o lançamento do ônibus espacial Endeavour uma transformação radical ocorreu no imageamento terrestre. A Missão Topográfica por Radar do Ônibus Espacial, mais conhecida pela sigla em inglês SRTM ${ }^{5}$, gerou um gigantesco arquivo de dados (12 terabytes) sobre $80 \%$ da massa de terra do planeta. Esta missão projetada e financiada pela Agência Espacial Americana $\left(\mathrm{NASA}^{6}\right)$, Agência Americana de

\footnotetext{
${ }^{4}$ Scientific Software Group (SSG-Surfer.com) - EUA

${ }^{5}$ Shuttle Radar Topography Mission

${ }^{6}$ National Aeronautics and Space Administration - EUA
} 
Inteligência Aeroespacial $\left(\mathrm{NGA}^{7}\right)$, em colaboração com a Agência Espacial Italiana $\left(\mathrm{ASI}^{8}\right)$ e o Centro Aeroespacial Alemão $\left(D^{2} R^{9}\right)$ permitiu o acesso a informações do relevo da Terra, com resolução de 90m para qualquer usuário, democratizando de maneira única dados até então desconhecidos de grandes porções do território, como por exemplo, da região amazônica, cuja dificuldade de mapeamento é notória devido a intensa cobertura de nuvens durante a maior parte do ano.

Na produção cartográfica, individual ou de grupos de pesquisa, notamos mudanças ao longo do período, com acesso aos computadores pessoais e depois os notebooks nos permitiram desenvolver trabalhos em períodos cada vez mais curtos, afinados com os crescentes avanços dos sistemas digitais que serviram de base para nossa produção científica no período.

A formulação de modelos matemáticos, o desenvolvimento de rotinas de mapeamento, a interpretação convencional por meio, por exemplo, de pares estereoscópicos e chaves de interpretação, são rapidamente suplantadas pelo acesso a computadores cada dia mais rápidos, com alta capacidade de memória, associados a programas computacionais e fontes de dados de sensoriamento remoto com resolução crescente.

Passamos rapidamente de desenvolvedores de mapas analógicos, para desenvolvedores de mapas decorrentes de rotinas semi-automáticas, muitas das quais desenvolvidas pelo próprio pesquisador, para usuários de sistemas computacionais como os CAD específicos para mapeamento, como por exemplo, o AutoCAD MAP, assim como de Modelos Numéricos do Terreno (MNT) e os Sistemas de Informação Geográfica (SIG).

No contexto brasileiro, assim como no mundial, o desenvolvimento na área de Cartografia, como nas demais áreas do conhecimento, tem início em ações acadêmicas e de grupos de pesquisa, normalmente vinculados a órgãos governamentais civis ou militares, passando em seguida para a iniciativa privada, de acordo com os interesses comerciais e a capacidade de mercado dos produtos gerados. Muitas dessas empresas têm, a sua frente, profissionais da academia que se associavam e/ou fundavam suas próprias empresas para comercialização de tais sistemas.

Hoje temos acesso a uma quantidade imensa de informação graças às pesquisas pioneiras dos militares americanos, na década de 1960, que buscavam criar pacotes de arquivos que

\footnotetext{
${ }^{7}$ National Geospatial-Intelligence Agency - EUA

${ }^{8}$ Agenzia Spaziale Italiana - Itália

${ }^{9}$ Deutschen Zentrums für Luft- und Raumfahrt (DLR) - Alemanha
} 
descentralizassem a informação contida no Pentágono por meio de comunicação digital. 0 interesse mundial aliado ao interesse comercial, rapidamente proporcionou a popularização da Internet na década de 1990, com bilhões de usuários no mundo.

Com a internet surgiram várias iniciativas de empresas para realizar pesquisa em seus bancos de dados, mas nenhuma com tanta repercussão como a Google Inc., fundada em 1998, com abertura para o publico em 2004, uma multinacional dos EUA que hospeda e desenvolve uma série de serviços e produtos baseados na Internet e gera lucro principalmente através da publicidade. A missão declarada da empresa desde o início é "organizar as informações do mundo e torná-las mundialmente acessíveis e úteis" (GOOGLE, 2014). Dentre as ferramentas disponiveis, estão o Google Earth e o Google Mapas, provavelmente os mais significativos serviços gratuitos de pesquisa e visualização de mapas e imagens de satélite da Terra pela internet.

O Google Earth apresenta modelos tridimensionais do globo terrestre e de outros corpos celestes, construídos a partir de mosaico de imagens de satélite, imagens aéreas, com recursos de 3D.

Já o Google Mapas possui ferramentas de visualização de mapas e imagens orbitais da Terra, também com recursos de visualização horizontal (street view) e 3D. Embora com uma defazagem temporal, que pode variar de 2 a 4 anos, dependendo da região da Terra, este dispositivo de pesquisa transformou radicalmente o uso dos mapas por pesquisadores e usuários comuns. Trata-se de um sistema que, de uma forma definitiva, vem democratizando a informação espacial, tornando-a acessivel em termos globais. $\mathrm{O}$ acesso aos mapas e imagens disponiveis por meio do Google Mapas permitem consultas dinamicas que solucionam muitos dos problemas corriqueiros de orientação e análise de mapas e imagens, antes de dificil acesso para o usuário não especialista. Além disso, o usuário pode intervir programando rotinas, inserindo informações cartográficas nos mapas existentes, valorizando aspectos desconhecidos através de imagens, fotografias horizontais e registros pessoais ou coletivos dos lugares destacados. Isso faz com que a Cartografia, cada vez mais faça parte do cotidiano das pessoas e permite produções cartográficas não convencionais, elaboradas por ações individuais ou comunitárias.

Tais fragmentos históricos, apresentados aqui de forma panorâmica, nos permitem refletir sobre a trajetória da Cartografia constantemente afinada com os principais avanços tecnológicos nos períodos, fazendo com que a ciência cartográfica se consolide em termos 
técnicos e metodológicos. A Cartografia, gradualmente, em termos nacionais e mundiais, continuará a ser conduzida pelos órgãos de mapeamento institucionais, compartilhe essa produção com iniciativas empresariais e individuais, na busca de soluções rápidas para problemas de planejamento e de estudos relacionados ao ambiente urbano e rural.

\section{Neocartografia: nossos mapas nunca mais serão os mesmos?}

Nos anos recentes temos observado, no meio acadêmico, uma nova tendência denominada por muitos de Neocartografia ou Nova Cartografia. Embora esse tipo de rotulação seja inadequado quando se considera a história e a evolução da ciência cartográfica, cujos fragmentos foram apresentados no item anterior, nos quais, a cada momento, a cartografia se transforma apoiada nas tecnologias, que são rapidamente suplantadas por outras novas e mais sofisticadas tecnologias ou seja, o novo de hoje seguramente é o velho de amanhã. Mesmo considerando a inadequação terminológica mas, na falta de outro termo que melhor exprima tal movimento, optamos nesse texto por apresenta essa tendência da Cartografia como vem sendo difundida pelos principais órgãos mundiais.

A Neocartografia se caracteriza por envolver a produção e o acesso aos documentos cartográficos por meio de dispositivos digitais como navegadores de internet, dispositivos de telefonia móvel, dentre outros. Configura-se como uma evolução da cartografia tradicional que está diretamente relacionada ao advento desses novos dados e interfaces, chamada de cartografia da internet social $^{10}$, conforme apresenta Turner (2013). O autor considera que as formas de materialização desses documentos cartográficos usualmente apoia-se na visualização por meio de mapas de internet, dos quais o mais conhecido e popularizado é o programa Google Mapas. Via de regra, tais recursos passam pela marcação de locais associados às mensagens, visualizações bidimensionais ou tridimensionais agregadas ou animações temporais:

Cartographically most visualizations apply traditional techniques of place marking these social messages, or performing limited aggregate visualization or perhaps temporal animation. Turner (2013, p.53).

\footnotetext{
${ }^{10}$ cartography of the social web
} 
O delineamento dessa tendência em linha de pesquisa é recente, cujos primeiros passos estão diretamente vinculados à criação da rede mundial de computadores, ou internet, que teve maior popularização a partir da abertura do Google Mapas em 2004.

A velocidade com que tal linha de pesquisa tem ganhado espaço em termos científicos se reflete na Comissão de Neocartografia da Associação Cartográfica Internacional (ICA), instituída durante a 25a Conferencia Cartográfica Internacional (ICC) realizada em Paris no ano de 2011. No ano de 2012 ocorreu em Londres o primeiro evento reunindo participantes da referida Comissão.

Existem muitas possibilidades de integração de novas tecnologias na Neocartografia, em várias modalidades das artes, jogos e videogames, aplicativos de celulares, dentre outros.

Os disseminadores da Neocartografia são chamados de Neocartógrafos, que Chilton (2013) caracteriza como:

The term neocartographers is being used to describe map makers who may not have come from traditional mapping backgrounds and are frequently using open data and open source mapping tools.(Chilton, 2013, p.52).

Segundo o autor, tais elaboradores de mapas não podem não ter o conhecimento vindo da cartografia tradicional e frequentemente apoiam seus produtos em ferramentas de mapeamento não comerciais, programas livres ou de código aberto ${ }^{11}$.

Como se trata de uma área ainda em formação, poucos são os artigos que detalham experiências envolvendo essas formas alternativas de mapeamento, visualização e interação com mapas em meio digital utilizando a nomenclatura Neocartografia.

Destacamos aqui alguns exemplos de Neocartografia recentemente divulgados e relacionados à criação de aplicativos (APP), apresentação de slides baseadas em mapas e estudo que trata da avaliação das tecnologias de mapeamento na internet.

Na primeira experiência, os autores tratam de um tema muito difundido nessa nova geração de celulares apoiados principalmente nos sistemas operacionais Android (Google Inc.), iOS (Apple Inc.), WP ${ }^{12}$ (Microsoft), que cada vez mais apresentam opções de programas aplicativos do tipo APP em telefones móveis e tablets com objetivos cartográficos de orientação e quantificação da informação.

Um exemplo apresentado na Conferencia Cartográfica Internacional realizada em Dresden Alemanha, no ano de 2013 diz respeito ao APP desenvolvido para detectar número de

\footnotetext{
${ }^{11}$ Open Source Software (OSS)

${ }^{12}$ Windows Phone
} 
pessoas que compõem uma multidão, denominado mapeador móvel de multidões ${ }^{13}$, apresentado por Krisp e Cheung (2013). O aplicativo faz uso de dispositivo de detecção de faces de indivíduos sendo a densidade calculada pela função de densidade Kernel (Kernel Density Estimation - KDE). O dispositivo permite a elaboração de representação temática de intensidade destacando a menor ou maior concentração de indivíduos por cores, que vão do azul ao vermelho, e sobrepõem a imagem capturada por meio de dispositivo móvel, como um celular.

Entendemos a experiência como promissora, no sentido de produzir resultados em tempo real, cujos mapeamentos de monitoramento de multidões podem ser adotados em eventos de grande porte, por meio de centrais de controle dessas aglomerações, auxiliando as ações de segurança pública. Vislubramos também o potencial de aperfeiçoamento do aplicativo, por exemplo, na pecuária, para o monitoramento de rebanhos de animais. É inquestionável a vantagem dessa extensão de recursos cartográficos para a segurança publica e o setor produtivo, que permitirá uma ampliação da cartografia como tecnologia que atenda às demandas sociais e econômicas.

Um outro exemplo de estudo de Neocartografia trata de investigação sobre a utilização das tecnológicas voltadas para a cartografia por parte de usuários especialistas de uma Universidade que, tradicionalmente atua no desenvolvimento de produtos e estudos apoiados em geotecnologias.

O estudo foi realizado por Roth et al. (2013) na Universidade de Wiscosin - EUA por meio de inquérito sobre avaliação de tecnologias de mapeamento emergentes na internet, mediante a análise da performance de programas e das preferências do usuário em termos de soluções para mapeamento. Como parte dos resultados das pesquisas realizadas, no que se refere a acesso a mapas da internet, pode-se concluir que somente o Google Mapas foi utilizado pela maioria (11 em um total de 21 participantes). Destacaram ainda que, quando procuram uma solução de mapeamento na internet, a principal característica para a seleção é a interatividade, seguida de facilidades de interface e possibilidades de múltiplas escalas, sendo a última referente aos recursos de animação. Como resultado do estudo os autores apresentam uma questão importante, que envolve a Neocartografia, quando consideram que quanto mais flexíveis as opções de mapeamento pela internet, mais difícil manter a fluência no uso e ensino dessas tecnologias. No nosso entendimento, a dinamicidade das

\footnotetext{
${ }^{13}$ mobile crowd mapper
} 
mudanças faz com que o usuário especialista não consiga acompanhar a velocidade de oferta e transformação das ferramentas disponíveis nessa área.

De acordo com os resultados do estudo e com base na nossa experiência acadêmica, observamos uma tendência à uniformização das fontes de dados da Cartografia na internet, predominando soberana a Google Inc. na veiculação de mapas e imagens que são utilizados tanto pelo usuário comum quanto pelos especialistas, bem como a dificuldade em acompanhar o volume de informação disponível para própria atualização de conteúdo, domínio das técnicas e divulgação por meio do ensino de tais tecnologias.

Outro exemplo de produção cartográfica fora dos padrões convencionais diz respeito às apresentações de slides baseadas em mapas ${ }^{14}$, que combina programa editor de slides com mapas, fotografias e textos escritos e narrados. Cartwright (2004) sugere o conceito de contar uma história geográfica, que geralmente corresponde a uma narrativa na forma de vídeo-documentário. Os autores Fujita; Arikawa (2012) apresentam estudo nessa perspectiva, no qual identificam e caracterizam histórias baseadas em mapas e a analisam a sua efetividade de edição numa perspectiva espaço-temporal. No artigo são apresentados fundamentos básicos e exemplos de roteiros de apresentações de slides espaciais criados pelo usuário, que podem também ser aplicados em outras atividades relacionadas à edição de mapas, como por exemplo, os vídeos elaborados por meio do Google Earth. Os autores detalham o processo de criação da apresentação de slides de mapas que incluem a précoleta de dados relativos aos mapas a serem utilizados na apresentação, o tempo, a distância a ser coberta e a trajetória de cada apresentação de slide, bem como a sequência a ser adotada e os padrões de apresentação.

A combinação de diferentes mídias e recursos sensoriais amplia a percepção e é amplamente explorada na Cartografia Escolar e em projetos que envolvem o ensino da Cartografia e a inclusão de pessoas com necessidades educacionais especiais, como é o caso, por exemplo, da Cartografia Tátil.

A experiência de Ventorini; Freitas (2011) apresenta o potencial da inclusão de dispositivos sonoros em maquetes e mapas táteis, permitindo aos alunos cegos e com baixa visão um melhor desempenho na interação com documentos cartográficos táteis, bem como na representação cartográfica de ambientes próximos como, por exemplo, o desenho da sala de aula.

\footnotetext{
${ }^{14}$ Map-Based Slideshow
} 
Observamos resultados positivos também na aplicação da Didática Multissensorial em estudos de geografia e cartografia, a qual faz uso de diferentes recursos que estimulam os sentidos como tato, visão, audição, paladar em atividades integradas que visem a exploração de um tema como estímulo ao aprendizado.

A busca interativa, a localização espacial e a obtenção de informações relacionadas aos lugares, possivelmente na forma multimídia, podem conduzir a uma melhor compreensão das inter-relações entre os objetos representados no mapa ou na maquete. (VENTORINI; FREITAS, 2011, p.63)

Uma outra experiência também confirma tal fato, divulgada por Juliasz; Freitas e Ventorini (2011), em estudo que trata da aplicação da Didática Multisensorial visando a elaboração e aplicação de coletânea de mapas táteis associados a sistemas sonoros e áudios que detalham a história e as tradições africanas e sua influência no Brasil. Ao apresentarem os resultados do trabalho as autoras argumentam:

Em relação à Didática Multisensorial, constatou-se a importância de recursos que estimulem todos os sentidos, para motivar os alunos a participarem ativamente das atividades, Uma das opções foi agregar nas representações espaciais as informações sonoras referentes à cultura afrobrasileira, como música, sotaque e relatos de pessoas descendentes dos quilombos. (JULIASZ, FREITAS e VENTORINI, 2011, p.217)

O uso de recursos como as apresentações de slide espaciais, bem como aqueles da didática multissensorial, que combinam mapas, fotografias, textos e narrativas orais têm amplo potencial para romper as barreiras da cartografia tradicional, apoiada nos mapas analógicos ou digitais estáticos, ampliando assim suas possibilidades sensoriais. Contribuímos com o entendimento e aprendizado do leitor/usuário ao combinarmos sequências espaciais ou temporais de mapas com sequências de imagens fotográficas formando pequenas animações dos locais registrados, acompanhados de textos elucidativos e de narrativa oral da história espacial a ser contada.

Com isso é possível alterar o relacionamento do usuário com os mapas, permitindo uma maior compreensão de seu conteúdo e, principalmente, de sua essência que é a expressão da realidade espacial de uma região da Terra.

\section{Considerações sobre Cartografia e as novas tecnologias}


Com base nos relatos aqui apresentados observamos o quão longa foi a evolução da Cartografia tradicional, para lançar as bases da produção cartográfica sistemática e temática em termos mundiais e nacionais, em contraponto com a rapidez e capacidade de disseminação e transformação da internet nas ciências em geral e, principalmente, no relacionamento do usuário comum com os mapas.

A preocupação que compartilhamos neste artigo é relativa à dificuldade das instituições acadêmicas e dos Institutos de Pesquisa e Desenvolvimento em Cartografia, de acompanhar, no que tange aos aspectos metodológicos e técnicos, este acelerado processo de popularização da informação geográfica visando disponibilizar à sociedade alternativas para a adequada comunicação cartográfica de tais documentos, bem como na solução de problemas decorrentes de erros ou imprecisões relacionados a escala, legenda e padronização cartográfica.

Muito mérito tem as iniciativas empresariais, com sistemas para integração de dados e de ferramentas de programação que se encontram disponíveis, permitindo a criação de mapas, roteiros e rotas personalizados, tendo como base os produtos cartográficos como os da Google Inc.. A facilidade com que se tem acesso aos dispositivos de busca espacial como, por exemplo, o Google Mapas e a abertura de tais sistemas para integração de informações pelo próprio usuário, permitem a ampliação de possibilidades de representação e comunicação cartográfica de forma criativa, fazendo com que algumas dificuldades ocorram no controle da qualidade da cartografia da internet. No caso específico da Google Inc., citado aqui como um dos mais populares programas de consulta e orientação por mapas e imagens, que tem especialistas em Cartografia contratados e também voluntários para realizarem o controle de qualidade, bem como a inserção de informações no sistema, um fator complicador é o volume da informação em termos globais e a velocidade com que tais mapas são atualizados por usuários, o que dificulta o controle de qualidade.

Uma outra preocupação que se apresenta decorre do grande volume de dados mundiais, assim como seu acesso e disponibilidade, estarem prioritariamente sob o controle da iniciativa privada e não de orgãos ou associações responsáveis pela produção cartográfica de países e continentes. O que hoje é o que melhor se aproxima de produto e ação democrática, pode se transformar em mecanismo de aprisionamento da informação, caso se alterem as políticas hoje vigentes à frente das empresas detentoras das chaves de acesso aos bancos de dados disponiveis e às tecnologias de desenvolvimento dos sistemas. 
Respondendo a pergunta apresentada no título desse artigo, nossos mapas sim, serão os mesmos no que se refere ao rigor cartográfico de apresentação, escala e generalização cartográfica, adoção das mais adequadas projeções e no controle de campo, legenda e respeito às normas de precisão e acuracidade expressas no Padrão de Exatidão Cartográfica (PEC) em vigor no Brasil.

Porém, nosso relacionamento com os mapas, a partir do advento da internet e dos dispositivos móveis como celulares e tablets, nunca mais será o mesmo. A velocidade com que a informação se torna acessível e a diversidade de programas computacionais de Cartografia, sejam comerciais ou livres, faz com que os usuários especialistas tenham dificuldades em acompanhar as transformações, se manter atualizados e permitir a adequada divulgação de seu uso por meio de atividades de ensino.

Os especialistas da área de Cartografia e Geotecnologias devem investir na adequação de sua produção cartográfica às novas possibilidades de multimídia e internet que se colocam disponíveis nos atuais dispositivos computacionais, visando ampliar o alcance do que vem sendo produzido nessas áreas nas instituições de ensino e pesquisa, sem deixar de lado o rigor que norteia a Cartografia. Também são responsáveis por investir em padrões de normatização das novas formas de produção cartográfica, orientar e avaliar a qualidade dos mapas produzidos por usuários não formados na Cartografia Tradicional, de modo a garantir o rigor e a qualidade na comunicação cartográfica e na qualidade de tais documentos. Com isso permitirão que a Cartografia Tradicional, Analógica, Digital, Social, Moderna, Pós Moderna, Neocartografia, e tantas outras possíveis denominações convirjam para uma única Cartografia, aquela que é base para nossa produção sistemática e temática, que preza pela fidelidade de localização, adotando um sistema de projeção adequado, numa dada escala, com base em diversificado arcabouço teórico-metodológico e técnico que permitem a fidedígna representação de objetos e fenômenos da superfície terrestre e de outros corpos celestes.

\section{REFERÊNCIAS}

CAMARA, G. A Pesquisa Espacial no Brasil: 50 anos de INPE (1961-2011). Revista USP. São Paulo, 89, p. 234-243, 2011. 
CARTWRIGHT, W. E. Exploring and Discovering Geographical Facts Using Geographical Storytelling. In: Anastasiou, P. (Editor); Trist, K. (Editor). Image, Text and Sound 2004: The Yet Unseen: Rendering Stories. Melbourne: RMIT Publishing, 2004: p.207-226. Disponível em: <http://search.informit.com.au/documentSummary;dn=875456614532907;res=IELHSS>. Acesso em: 12 Jun 14.

CHILTON, S. Is there a new cartography? In: INTERNATIONAL CARTOGRAPHIC CONFERENCE (ICC): From pole to pole, $26^{\text {th }}$, 2013. Dresden. Proceedings... Dresden: ICA, 2013. p. 53 (\#246)

DOYLE, F. Digital Terrain Models: An Overview. Photogramnetric Engineering and Remote Sensing. Bethesda, 44(12), p. 1481-1485. 1978.

FUJITA, H.; ARIKAWA, M. A User Study of a Map-Based Slideshow Editor. Cartographica. Toronto, 46:2, p. 74-82. 2011.

GOOGLE. Empresa Google. 2014. Disponível em: <https://www.google.com/intl/ptBR/about/company/>. Acesso em: 12 jun. 2014.

JULIASZ, P. C. S.; FREITAS, M. I. C.; VENTORINI, S. E. Cartografia Tátil e Didática Multisensorial: Uma construção diferenciada de mapas sobre a África e sua influência no Brasil. In: Freitas, M. I. C.; Ventorini, S. E. (Org.). Cartografia Tátil: Orientação e mobilidade para pessoas com deficiência visual. Jundiaí: Paco Editorial, 2011. p. 193-217.

KRISP, J.; CHEUNG, A. Mobile Crowd Mapping. In: INTERNATIONAL CARTOGRAPHIC CONFERENCE (ICC): From pole to pole, $26^{\text {th }}, 2013$. Dresden. Proceedings... Dresden: ICA, 2013. p. 1-9 (\#106)

ROTH, R. E.; DONOHUE, R. G.; SACK, C. M.; WALLACE, T. R.; BUCKINGHAM, T. M. A. A Process for Assessing Emergent Web Mapping Technologies. In: INTERNATIONAL CARTOGRAPHIC CONFERENCE (ICC): From pole to pole, $26^{\text {th }}, 2013$. Dresden. Proceedings... Dresden: ICA, 2013. p. 1-15. (\#380)

TURNER, A. Neocartography and the Social Web In: INTERNATIONAL CARTOGRAPHIC CONFERENCE (ICC): From pole to pole, $26^{\text {th }}, 2013$. Dresden. Proceedings... Dresden: ICA, 2013. p. $53(\# 1218)$ 
VENTORINI, S. E.; FREITAS, M. I. C. Representação espacial e ausência de visão: Relato de Experiência. In: Freitas, M. I. C.; Ventorini, S. E. (Org.). Cartografia Tátil: Orientação e mobilidade para pessoas com deficiência visual. Jundiaí: Paco Editorial, 2011. p. 33-60. 